\title{
Addiction: The Dark Side of Learning
}

$\mathrm{C}$ hildren have an amazing facility to learn, and our society does everything to encourage this. Learning is good and it is difficult to conceive that too much could have a negative impact. However, this is just what recent theories on addiction suggest; addictive substances promote the learning of drugrelated behaviors such that they become exclusive. As a consequence drug-use becomes compulsive and continues despite negative consequences, a defining feature of addiction. This theory has its roots in the assumption that dopamine released by the neurons of the midbrain represents a strong learning signal.

Under physiologic conditions, midbrain dopamine neurons are activated by natural rewards, such as food and sex, which are crucial for the survival of the species. For the subject, it is therefore important to learn the circumstances under which rewards are obtained. This includes rapid acquisition of both predictive cues and efficient behaviors that are successful in obtaining rewards.

When the activity of midbrain dopamine neurons is monitored in animals in vivo, unexpected rewards induce an increase of the firing rate leading to the release of dopamine in the nucleus accumbens and the prefrontal cortex. Conversely, predicted rewards do not increase dopamine levels. Thus, the brain estimates and holds in memory the utility of possible actions, based on the reward that has been obtained in the past. The actual reward is then compared with this prediction. The difference - also called the prediction error of reward - facilitates learning of behaviors that maximize reward. Eventually, after several iterations of this process, the animal's behavior is optimized to obtain the reward with certainty. The reward prediction error therefore tends to zero and dopamine neurons are no longer activated when the reward is obtained.

With addictive drugs this resetting of the prediction error signal fails. In fact, it is a defining commonality of addictive drugs that they increase mesolimbic dopamine. In contrast to natural reward, this increase occurs with each intake and is much stronger. All happens as if the brain could never predict the reward, drugs always appear better than expected (1). The learning therefore never ends, which eventually leads to compulsion even when the pleasure is long gone: wanting without liking.

On the cellular level there is a myriad of recent studies that indicate that inappropriate release of dopamine in response to drugs causes long-term alterations of synaptic transmission in the mesolimbic dopaminergic system. Drugs of abuse induce synaptic plasticity that might represent the cellular substrate of pathologic learning observed in addicts. Such pathologic plasticity has been observed in many parts of the brain, and starts with a potentiation of excitatory synapses formed onto dopaminergic neurons in the midbrain hours after a single injection of cocaine. This plasticity involves a redistribution of glutamate receptors of the AMPA type such that they become calcium permeable (2), which may trigger downstream signaling events eventually leading to addiction.

As radical as these changes are, they do not lead to addiction in every subject exposed. Even with prolonged recreational cocaine use only 1 out of 5 will eventually be diagnosed with the disease. This individual vulnerability is still poorly understood and may involve genetic as well as environmental factors. In addition, for a given individual, the risk to become addicted varies during development, which leads to the notion of "critical periods." In the pediatric population, for example, exposure to drugs in utero or during adolescence is associated with an increased liability of abuse in adulthood. It will therefore be of great interest to examine how the exposure to drugs in such situations interferes with the high degree of plasticity in the brain of children. - Bénédicte Balland and Christian Lüscher

\section{REFERENCES}

1. Redish AD 2004 Addiction as a computational process gone awry. Science 306:19441947

2. Mameli M, Balland B, Luján R, Lüscher C 2007 Rapid synthesis and synaptic insertion of GluR2 for mGluR-LTD in the ventral tegmental area. Science 317:530 533 